\title{
Preparation of Multicore Colloidosomes: Nanoparticle-Assembled Capsules with Adjustable Size, Internal Structure, and Functionalities for Oil Encapsulation
}

\author{
David F.F. Brossault ${ }^{1,2}$, Thomas M. McCoy ${ }^{1,2}$ and Alexander F. Routh ${ }^{1,2 *}$ \\ 1. Department of Chemical Engineering and Biotechnology, University of Cambridge, Philippa \\ Fawcett Dr, Cambridge CB3 OAS, United Kingdom \\ 2. BP Institute, University of Cambridge, Madingley Rise, Cambridge CB3 OEZ, United Kingdom \\ E-mail: afr10@cam.ac.uk
}

\begin{abstract}
Colloidosomes, also known as Pickering emulsion capsules, have attracted attention for encapsulation of hydrophilic and hydrophobic actives. However, current preparation methods are limited to single core structures and require the use of modified/engineered nanoparticles for forming the shell. Here, we report a fast, simple, and versatile method for producing multioil core silica colloidosomes via salt-driven assembly of purely hydrophilic commercial nanoparticles dispersed within an Oil-In-Water-In-Oil (O/W/O) double emulsion template. The internal structure and overall diameter of the capsules can be adjusted by altering the primary and secondary emulsification conditions. With this approach, 7 to $35 \mu \mathrm{m}$ diameter multicore colloidosomes containing 0.9 to $4.2 \mu \mathrm{m}$ large oil cores were produced. The capsules can easily be functionalized depending on the type of nanoparticles used in the preparation process. Here, metal oxide nanoparticles, such as $\mathrm{Fe}_{3} \mathrm{O}_{4}, \mathrm{TiO}_{2}$ and $\mathrm{ZnO}$, were successfully incorporated within the structure, conferring specific functional properties (i.e. magnetism, photocatalysis) to the final microcapsules. These capsules can also be ruptured using ultrasound, enabling easy access to the internal core environments. Therefore, we believe this work offers a promising approach for producing multicore colloidosomes with adjustable structure and functionalities for the encapsulation of hydrophobic actives.
\end{abstract}

\section{Keywords}

Multicore colloidosome, Double emulsion, Nanoparticle, Self-assembly, Magnetism, Photocatalysis, Oil encapsulation. 


\section{Introduction}

Microencapsulation has attracted much research interest for protecting and delivering active ingredients in food and beverage, ${ }^{[1,2]}$ cosmetic and personal care, ${ }^{[3,4]}$ agrochemical ${ }^{[5,6]}$ and pharmaceutical ${ }^{[7,8]}$ applications. The method consists of the entrainment of a solid, liquid, or gaseous compound, called the active material, within another structure referred to as the shell, to ensure its protection, control its release or improve its taste, odor, or handling. ${ }^{[9]}$ Multiple encapsulation techniques have been reported using thermal, electrostatic, or chemical methods to produce core/shell capsules. ${ }^{[9,10]}$ Among them, colloidosomes appear as a promising system. Such capsules, originally reported by Velev and co-workers ${ }^{[11]}$ and named by Dinsmore et al. ${ }^{[12]}$, are composed of a gel or liquid core surrounded by a nanoparticleassembled shell. ${ }^{[13,14]}$ These superstructures offer high control on permeability, ${ }^{[15,16]}$ functionalities ${ }^{[17-19]}$ and release mechanisms, ${ }^{[20-24]}$ depending on the preparation method and choice of shell colloidal particles. Colloidosomes are conventionally produced via a two-step approach. First, a particle stabilized or Pickering emulsion, ${ }^{[25]}$ is formed via adsorption of colloidal particles at an oil/water interface. Particles must exhibit affinity for both the aqueous and organic phases, which often requires adjustment of particle wettability by the adsorption of additives (e.g. surfactants, polymers) or chemical functionalization. ${ }^{[26,27]}$ Particles are then locked at the interface, producing a robust microcapsule for utilization. ${ }^{[13,14]}$ Depending on the preparation method, colloidosomes can be used for encapsulation of hydrophilic or hydrophobic actives. Active materials such as yeasts, ${ }^{[28]}$ bacteria, ${ }^{[29]}$ enzymes, ${ }^{[30]}$ or therapeutic molecules [31] have been successfully encapsulated within water-core colloidosomes. Oil-core colloidosomes have also been explore to address the usual volatility or stability concerns inherent with hydrophobic actives (e.g. Flavors, fragrances, antioxidants, vitamins, pesticides, etc.). ${ }^{[32-44]}$

These oil-core systems are conventionally produced from a Pickering-emulsion template, with particles locked together in a robust shell through thermal annealing, ${ }^{[16]}$ layer-by-layer polyelectrolyte deposition, ${ }^{[33-35]}$ ionotropic gelation, ${ }^{[36,37]}$ cross-linking, ${ }^{[38-40]}$ interfacial polymerization ${ }^{[41]}$ or growing of an additional inorganic ${ }^{[42,43]}$ or metallic shell ${ }^{[44]}$. However, such approaches exhibit certain limitations. Firstly, the need for an intermediate Pickering emulsion step, requires the design/modification of nanoparticles to obtain suitable Pickering emulsifiers, raising the cost and complexity of the method. Secondly, the locking step may be 
time-consuming, hazardous and costly due to modification of experimental conditions (e.g. $\mathrm{pH}$, Temperature) or further addition of toxic chemicals to the system (i.e. Cross-linkers, polymers, polyelectrolytes), which may impact the stability of encapsulated actives. Finally, only single core systems have been reported, limiting such structures to single-step release mechanisms. Producing multicore colloidosomes could enable encapsulation of multiple hydrophobic cargoes, as well as a multi-stage release of actives. Contrary to polymeric multicore microcapsules, which have been reported at multiple times, multicore colloidosomes have seldomly been explored. To the best of the author's knowledge, such structures have only been reported by Lee and Weitz, with the preparation of non-spherical multi-compartment colloidosomes obtained from assembly of hydrophobic particles within a Water-in-Oil-in-Water (W/O/W) emulsion template. ${ }^{[45]}$ In their work, Lee and Weitz described the preparation of non-spherical multi-compartment colloidosomes obtained from assembly of hydrophobic particles within a Water-in-Oil-in-Water (W/O/W) emulsion template. However, this approach was limited to a water-core structure and required the use of a solvent evaporation approach for producing the capsules.

Here, we present a facile, safe, and adjustable method for producing spherical multicore colloidosomes via salt-driven assembly of commercial nanoparticles in an Oil-in-Water-in-Oil $(\mathrm{O} / \mathrm{W} / \mathrm{O})$ emulsion template. In this approach, capsules are prepared at room temperature without requiring an intermediate Pickering-emulsion template. The use of a colloidal destabilization approach as a locking mechanism makes this method environmentally benign. Furthermore, the internal structure and functionalities of the final capsules can be easily tuned by altering the mixing conditions and composition of the aqueous phase.

\section{Results and discussion}

Silica nanoparticles used in these experiments are strongly hydrophilic, inhibiting their adsorption at the oil/water interface for formation of Pickering emulsions. However, these particles can easily be destabilized, and aggregate upon addition of salt. When these particles were dispersed in ultrapure water, the $\mathrm{pH}$ increased slightly to values between 9 and 10 . In this $\mathrm{pH}$ range, the silica nanoparticles readily disperse and bear a strong negative surface charge measured between -40 and $-50 \mathrm{mV}$. Addition of calcium chloride induces surface charge screening and subsequent nanoparticle agglomeration. This hetero-coagulation 
approach has recently been reported in reverse emulsions for room-temperature production of magnetic silica microparticles $\left(\mathrm{Fe}_{3} \mathrm{O}_{4} / \mathrm{SiO}_{2}\right)^{[46]}$ and magnetic photocatalysts $\left(\mathrm{TiO}_{2} / \mathrm{Fe}_{3} \mathrm{O}_{4} / \mathrm{SiO}_{2}\right)^{[47]}$. Here, formation of an $\mathrm{O} / \mathrm{W} / \mathrm{O}$ emulsion serves to structurally template assembly of the capsules, with multiple oil cores embedded within their structure.

The major advantage of the present approach for capsule formation is that it circumvents the necessity for creation of a Pickering emulsion. This can be challenging to achieve, as the colloidal particles must have an inherent affinity for the oil-water interface. Such particles are often surface functionalised (ie. Janus particles) or incorporated with additives to facilitate interfacial adsorption (ie. Polymers or charged surfactants), making the system more complex and expensive. The current method allows for formation of capsules by aggregating purely hydrophilic commercial nanoparticles that are dispersed in the aqueous medium surrounding the surfactant stabilised oil droplets. Therefore, the nanoparticles can be used directly, without adjusting their wettability. The complete preparation method is shown in Scheme 1.

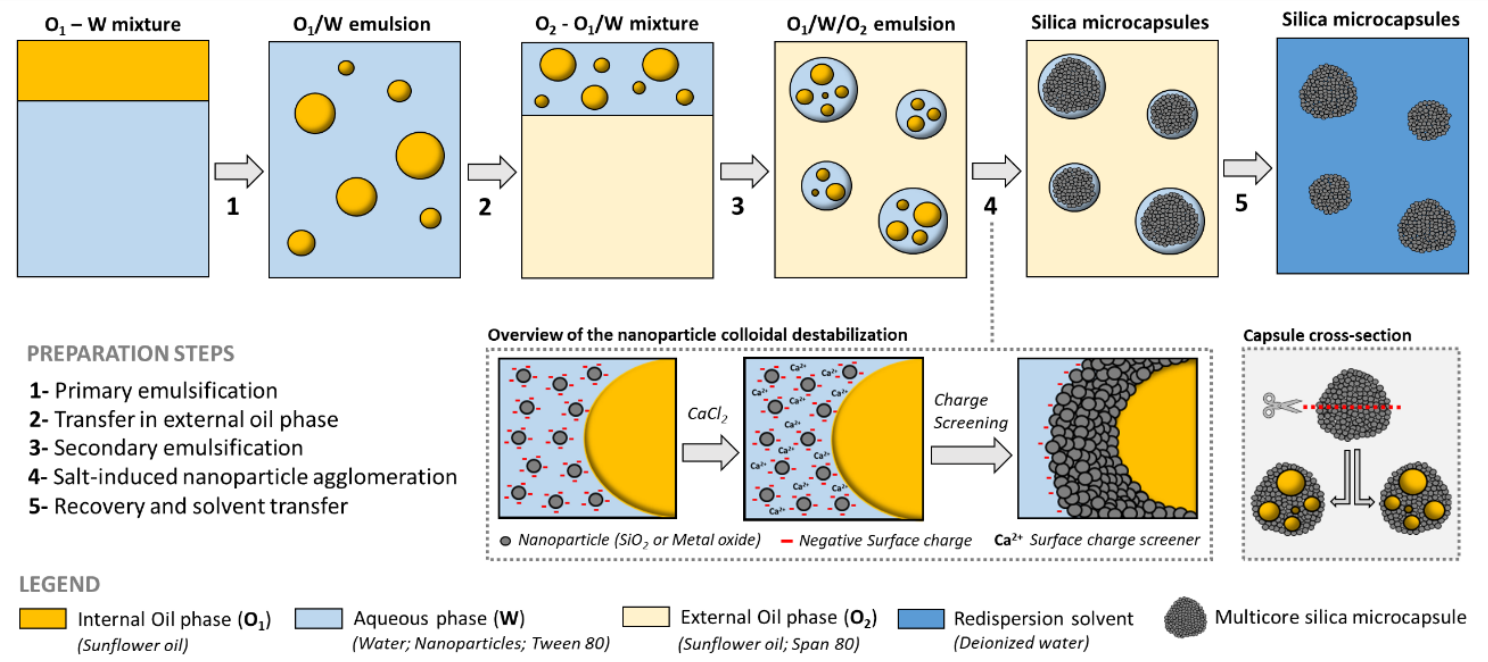

Scheme 1. General procedure for preparing multi-oil core silica microcapsules using commercial nanoparticles dispersed in an Oil-in-Water-in-Oil emulsion template.

\subsection{Preparation of multicore capsules with tunable core size and overall diameter}

In our preparation method, the capsule structure is directly controlled by utilisation of primary and secondary emulsions, with the former templating the inner cores and the latter templating the overall capsule size. Therefore, size and stability of these emulsions is crucial in the design of our capsules. Preliminary experiments were carried out to investigate the 
impact of mixing conditions and surfactant concentration on emulsion preparation (Figures S1 to S4). Results showed that primary $\left(\mathrm{O}_{1} / \mathrm{W}\right)$ emulsions prepared with $5 \mathrm{wt} \%$ Tween 80 were stable for more than $24 \mathrm{~h}$ and the diameter of the dispersed droplets could easily be adjusted between 0.9 and $4.2 \mu \mathrm{m}$, depending on the mixing conditions. Double $\left(\mathrm{O}_{1} / \mathrm{W} / \mathrm{O}_{2}\right)$ emulsions, prepared from those primary emulsions, were stable for $30 \mathrm{~min}$, enabling their use as microcapsule template.

The impact of primary emulsification parameters on the capsule internal structure was first assessed by producing capsules from double emulsions prepared at different primary emulsification conditions (Ultra-Turrax (UT) operating at $5,000,10,000$ or 15,000 RPM), and fixed secondary emulsification conditions (Vortex mixer (V) operating at 2,000 RPM). Microscopy images confirmed the production of spherical capsules with an average overall diameter of approximately $18 \mu \mathrm{m}$, regardless of mixing parameters (Figure 1). STEM and optical microscopy images confirmed the presence of multiple cores within the capsules and showed a significant decrease in internal core diameter with increased mixing speeds (3.3 to $0.8 \mu \mathrm{m})$. The size of these cores correlates with the diameter of oil droplets dispersed in the primary emulsions (Figure S1), confirming that primary emulsification conditions dictate the capsule internal structure. By increasing the energy input, primary emulsions with smaller oil droplets are formed and subsequently used to template smaller oil cores within the final capsule structure. Triplicated samples confirmed the repeatability of the procedure, with deviation in overall capsule and inner core diameters being less than $15 \%$. Low magnification optical microscopy images and size distribution histograms of those samples are provided in the Supporting Information (Figures S5 to S7). It is worth mentioning that the apparent discrepancy between the reported size values and the microscopy images shown in this article, originates from the small number of capsules effectively present on the image. Size distribution histograms, provided in supporting information, showed a Poisson distribution, with capsule diameter ranging from 5 to $50-60 \mu \mathrm{m}$. This polydispersity in diameter directly originates from the bulk emulsification approach used for the preparation of the capsule templates. 


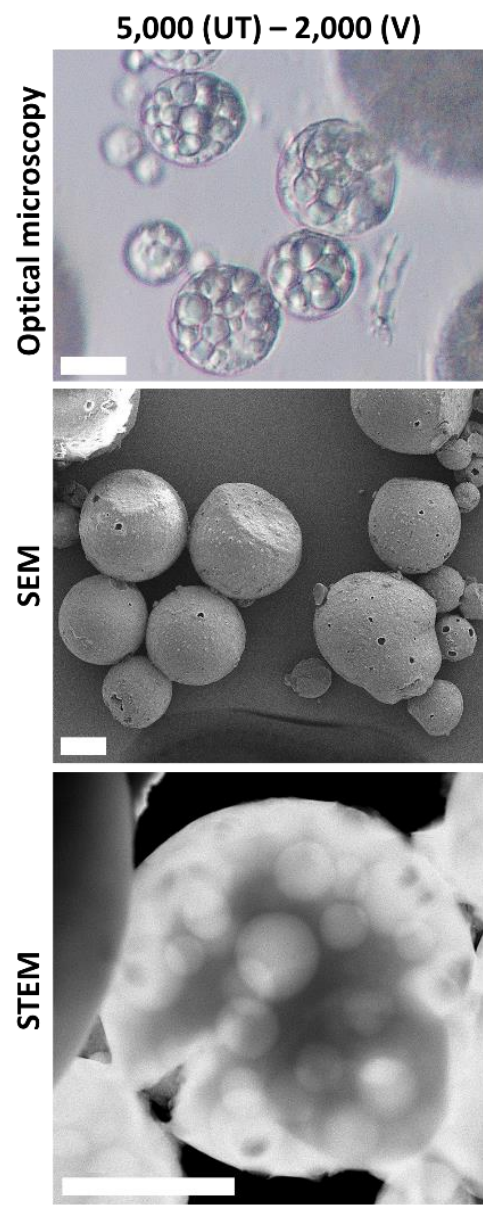

Core size: $3.3 \pm 0.8 \mu \mathrm{m}$

Capsule size: $17.4 \pm 9.6 \mu \mathrm{m}$
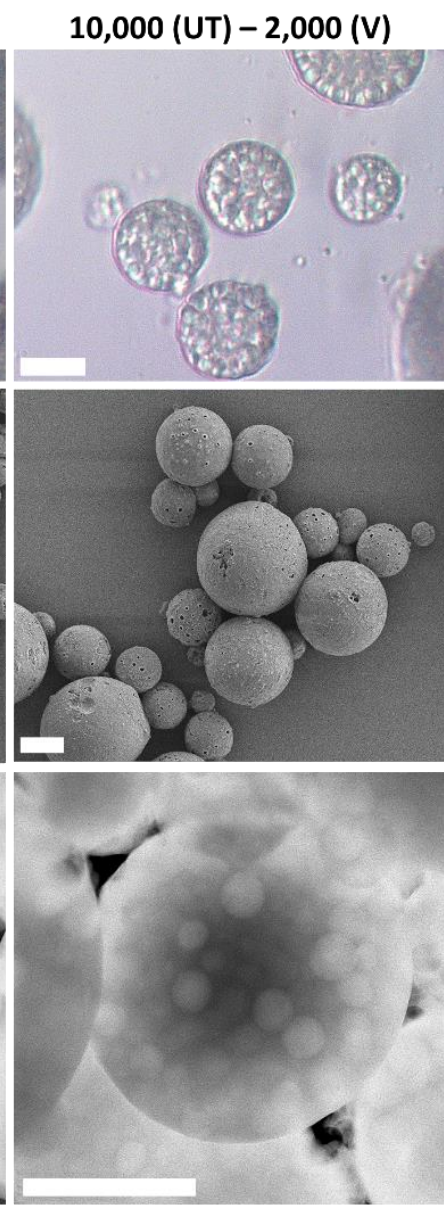

Core size: $1.5 \pm 0,4 \mu \mathrm{m}$

Capsule size: $18.3 \pm 7.8 \mu \mathrm{m}$
$15,000(U T)-2,000(V)$
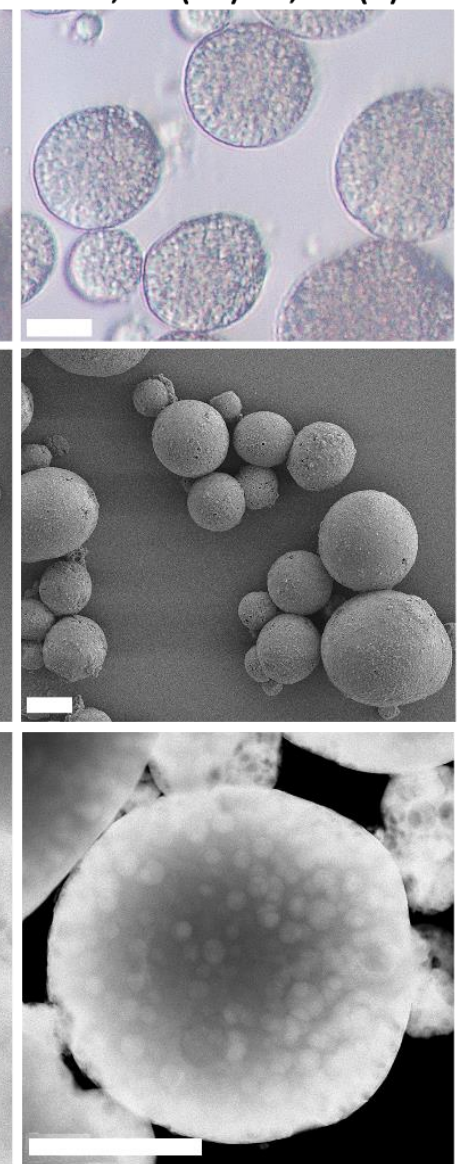

Core size: $0.8 \pm 0.2 \mu \mathrm{m}$

Capsule size: $17.9 \pm 9.0 \mu \mathrm{m}$

Figure 1. Microscopy images of multi-oil-core silica microcapsules prepared with different primary emulsification mixing speeds (shown at the top of the figure). Scale bars are equal to $10 \mu \mathrm{m}$. Average size values were obtained from the analysis of optical microscopy images $(\mathrm{N}=250)$.

The surface defects, observed by SEM (Figure 1), are likely to be due to the presence of oil cores adjacent to the double emulsion boundaries prior to capsule formation. In this condition, only a thin layer of aqueous phase is located between the internal oil core and the external oil phase limiting the amount of silica available for colloidal destabilization. This results in two possible situations. A thin silica layer may form upon colloidal destabilization. This thin shell may then break during the mixing process due to high shear stresses, exposing the surface pores. Alternatively, a silica depletion may occur in the thin aqueous layer. As the silica agglomerate grows within the aqueous phase the silica nanoparticles are attracted to the growing fractal structure. Because the silica aggregate occupies less volume than the corresponding aqueous phase there will be insufficient silica within the aqueous layer to form 
a complete shell, leaving behind surface pores. The oil is then removed from the cavities via coalescence with the external oil phase or during the cleaning stage. Additional SEM images suggest that the size and frequency of such defects can be significantly reduced by decreasing the diameter of the internal oil cores. This helps to ensure that they are more likely to locate completely within the capsule confines. An alternative way to minimize this phenomenon would be to reduce the overall amount of oil within the capsules, enabling enhanced diffusion of oil droplets within double emulsions prior to colloidal destabilization.

The impact of secondary emulsification parameters on overall capsule structure was then explored, by producing capsules from double emulsions prepared at different primary (UT operating at 5,000,10,000 or 15,000 RPM) and secondary (Manual mixing, V or UT operating at diverse speeds) emulsification conditions (Figure 2). A decrease in the overall capsule diameter was observed for samples prepared using the higher energy input technique (UT), and this was found to occur irrespective of primary emulsification conditions (Figure 2A-C). By increasing the energy input during the secondary emulsification step, smaller double emulsions are produced resulting in smaller capsules. Using this approach, multicore silica capsules with a mean diameter between 7 and $35 \mu \mathrm{m}$ can be produced. It is interesting to note that the average diameter of the capsules significantly decreases after modification of the mixing technique but appears to level off for increased mixing speed. This finding suggests an inefficiency in the mixing process, where the excess energy brought to the mixture is mostly converted into viscous losses rather than used for decreasing the diameter of the double emulsion template. Measurement of internal core diameters showed a significant impact of extreme secondary emulsification conditions on the capsule internal structure. This was evidenced by the decrease in core diameter measured for capsules prepared at high secondary emulsification speed (i.e. 10,000 or 15,000 RPM (UT)) (Figure 2D). At this secondary mixing speed, oil droplets are further dispersed within the double emulsion, resulting in a reduction of average core diameter from 2.8 to $0.8 \mu \mathrm{m}$. Therefore, a reduced secondary mixing speed (i.e. 3,000 or 5,000 RPM (UT)) should be exploited to produce small capsules with large oil cores. Optical microscopy images of all samples are provided in the Supporting Information (Figure S8). 

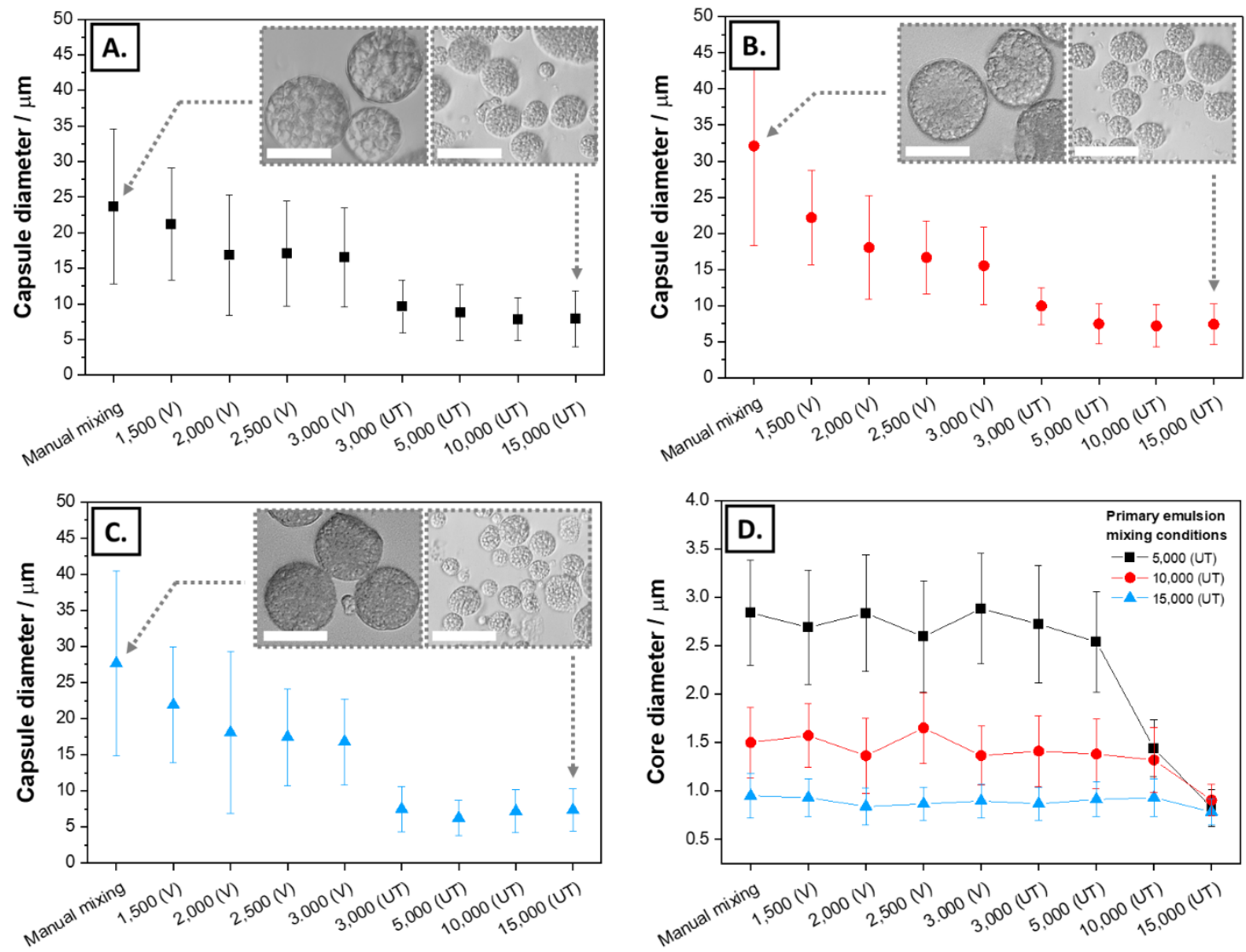

Figure 2. Impact of secondary emulsification mixing speed on (A-C) overall capsule diameter and (D) inner core diameter. Capsules obtained using primary emulsions prepared with an Ultra-Turrax operating at (A) 5,000, (B) 10,000 and (C) 15,000 RPM. Insets are optical microscopy images of capsules produced at the indicated mixing conditions. Scale bars are equal to $20 \mu \mathrm{m}$.

These results show that capsules produced using this method display a high degree of tunability, enabling tailoring of their internal structure and overall size by simply adjusting the mixing conditions.

\subsection{Facile shell functionnalization}

Metal oxide doped multicore colloidosomes were prepared via dispersion of $\mathrm{Fe}_{3} \mathrm{O}_{4}, \mathrm{TiO}_{2}$ or ZnO nanoparticles within the aqueous phase prior to capsule formation. EDS elemental mapping analysis confirmed the successful incorporation of nanoparticles within the capsule structure (Figure 3A-C). Optical microscopy images and size distribution histograms showed that this incorporation did not alter the size or structure of the final capsules prepared at 15,000 (UT) $-2,000(V)$, resulting in 17 to $20 \mu \mathrm{m}$ large capsules with $1 \mu \mathrm{m}$ cores. 

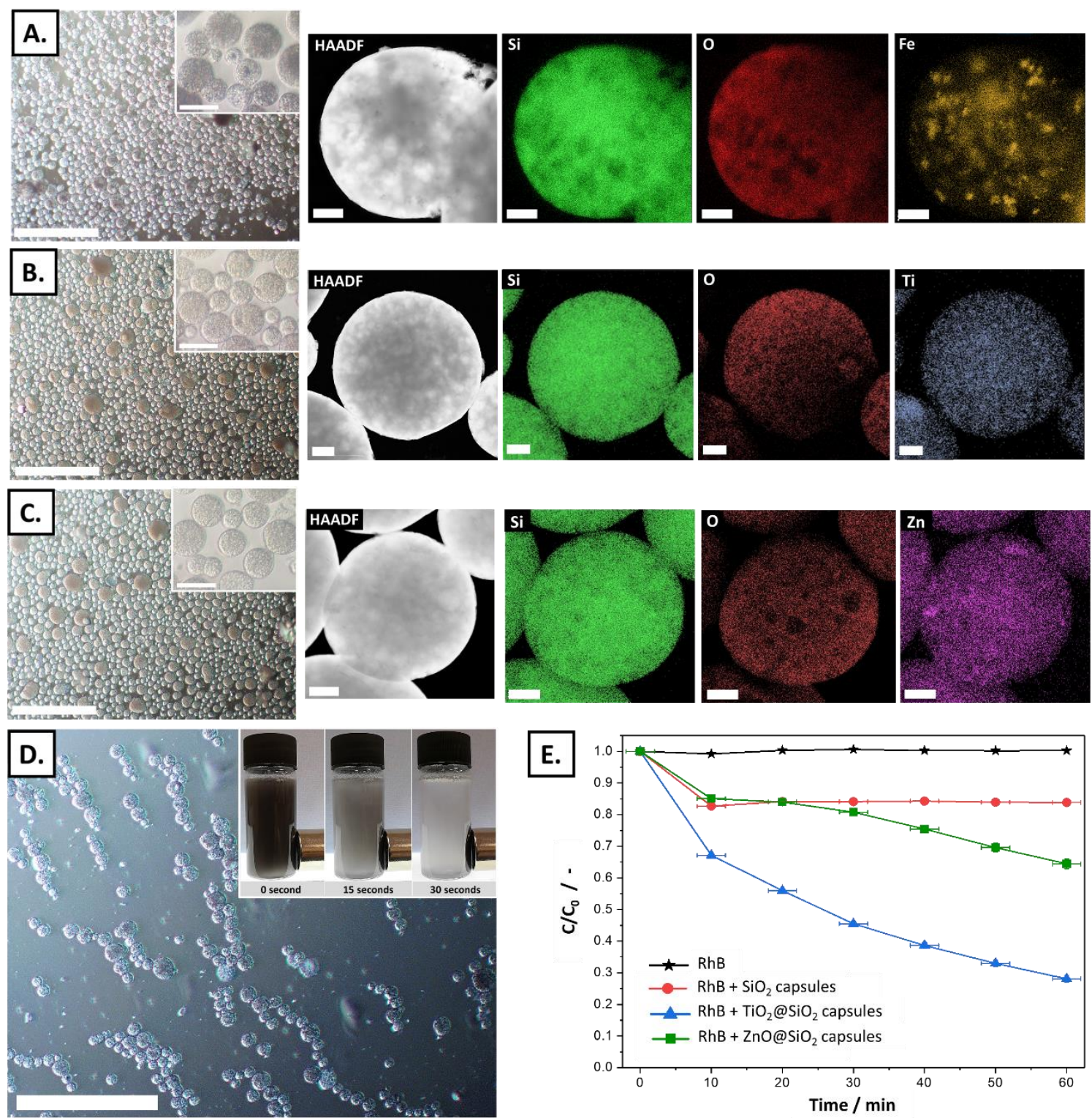

Figure 3. Characterization of metal oxide doped silica microcapsules $(15,000$ (UT) $-2,000$ (V)). Optical microscopy images and EDS elemental mapping of capsules doped with (A) $\mathrm{Fe}_{3} \mathrm{O}_{4}$, (B) $\mathrm{TiO}_{2}$ and (C) $\mathrm{ZnO}$ nanoparticles. (D) Response of $\mathrm{Fe}_{3} \mathrm{O}_{4}$ doped capsules to external magnetic fields. (E) Degradation of a Rhodamine B solution (50 mL, $\left.10 \mathrm{mg} . \mathrm{L}^{-1}\right)$ under UV-light irradiation in the presence of $\mathrm{TiO}_{2}$ and $\mathrm{ZnO}$ doped capsules (100 mg). Scale bars are equal to $200 \mu \mathrm{m}$ on optical microscopy images ( $20 \mu \mathrm{m}$ on inset images) and to $2 \mu \mathrm{m}$ on STEM images.

The incorporation of various nanoparticles within the capsule structure confers additional physicochemical properties to the system. For instance, $\mathrm{Fe}_{3} \mathrm{O}_{4}$-doped microcapsules were shown to be magnetically responsive, with their recovery from solution observed to be fewer than 30 seconds under an external magnetic field (Figure 3D). 
Photocatalytic properties of $\mathrm{TiO}_{2}$ and $\mathrm{ZnO}$ doped capsules were investigated by exploring the degradation of an aqueous solution of synthetic dye (Rhodamine B) upon UV-light irradiation. Results display enhanced dye removal for capsules integrating these metal oxides (Figure 3E). In the absence of capsules, minimal degradation of Rhodamine B under UV-light irradiation was observed, confirming the dye stability and importance of the photocatalytic materials. In the presence of multicore silica colloidosomes without metal oxide dopants, a $16 \%$ decrease in dye absorbance was observed after 1 hour of UV irradiation. Beyond 10 minutes, any additional irradiation does not result in further decreases in absorption, suggesting that the mechanism may be due to adsorption from solution rather than photodegradation. The capsules bear a negative surface charge at neutral $\mathrm{pH}$, and since Rhodamine $\mathrm{B}$ features a cationic quaternary ammonium group, this mechanism is feasible. The observed plateau is likely due to saturation of the capsule surfaces with Rhodamine $B$, preventing further removal from solution. In the presence of $\mathrm{TiO}_{2}$ doped capsules, a $72 \%$ decrease in the absorption maxima were observed after $1 \mathrm{~h}$ of UV irradiation. The corresponding result for $\mathrm{ZnO}$ doped capsules was $36 \%$. The steady decrease over time suggests that removal of Rhodamine B from solution is not only based on an adsorption mechanism, as observed with $\mathrm{SiO}_{2}$ microcapsules, but also on a photodegradation mechanism. Absorbance spectra in these cases exhibit both a decrease in the maximum absorbance as well as a hypsochromic shift, characteristic of degradation of the aromatic ring and de-ethylation of Rhodamine $B^{\text {[48-50] }}$.

This phenomenon is based on a well-known UV light-induced photoexcitation process. Under UV-light irradiation, electrons are transferred from a valence band to a conduction band within the $\mathrm{TiO}_{2}$ and $\mathrm{ZnO}$ nanoparticles incorporated within the capsules. The electron and hole pair generated react with $\mathrm{O}_{2}$ and $\mathrm{H}_{2} \mathrm{O}$ to subsequently form super-oxide species $\left(\mathrm{O}_{2} \cdot\right)$ and hydroxyl radicals $(\mathrm{OH} \cdot)$, which are then involved in the oxidation of the dye. The difference in degradation efficiency between the two systems is likely to arise from the lower amount of metal oxide dopant within the $\mathrm{ZnO}$ capsules compared to those with $\mathrm{TiO}_{2}$. Adjusting the loading of nanoparticles within the capsules as well as capsule diameter or light source conditions, would likely alter the degradation dynamics. It is also worth mentioning that the dye removal results presented here correspond to a combination of adsorption and photodegradation effects of the assembled capsules. A preliminary equilibration of the 
samples in the dark prior to UV-light irradiation could be implemented to assess the extent of each mechanism on the overall dye removal efficiency.

This approach enables facile, fast, and diverse capsule production and functionalization, without the need for nanoparticle surface modification or post-production capsule coating. This work has demonstrated the incorporation of $\mathrm{Fe}_{3} \mathrm{O}_{4}, \mathrm{TiO}_{2}$ and $\mathrm{ZnO}$ nanoparticles, although other materials (e.g. metal oxides, charged polymeric particles, carbon nanodots) could be included within the capsule structure, provided they can be homogeneously dispersed in the aqueous phase and destabilized upon addition of salt. Additional EDS mapping analysis, optical microscopy images, size distribution histograms and absorbance spectra related to these experiments are provided in the Supporting Information (Figures $\mathbf{5 9}$ to S11).

\subsection{Rupture of capsules with ultrasound}

Ultrasound was applied to multicore silica microcapsules to assess their structural integrity (Figure 4A). Microscopy performed before and after treatment provided key structural information (Figure 4B-E). The presence of oil cores within the capsules was easily confirmed by the difference in contrast observed between the embedded oil droplets and silica shell. After applying ultrasound, non-miscible droplets and shell fragments were observed in solution confirming capsule rupture and release of the hydrophobic contents (i.e. sunflower oil). Images obtained after increased ultrasound duration suggest a direct correlation between the capsule internal structure and robustness. Capsules with large cores were observed to break into non-spherical fragments upon ultrasonication, resulting in complete rupture of the capsules after $3 \mathrm{~min}$ of treatment (Figure 4B and 4D). Fragments observed following rupture did not show any apparent contrast within their structure, suggesting complete release of sunflower oil. Conversely, capsules with smaller cores remained spherical upon ultrasonication (Figure $4 \mathrm{C}$ and $\mathbf{4 E}$ ). Increasing the ultrasound duration appeared to result in superficial erosion with spherical capsules measured by optical microscopy to be 17,38 and $50 \%$ smaller after 1, 2, and 3 min of ultrasound. The contrast observed within the final capsules suggests the presence of residual oil cores, still available for further release steps. Observation of broken capsules via SEM confirmed the multicore structure with average inner core diameters of $2.8 \pm 0.5$ and $0.9 \pm 0.2 \mu \mathrm{m}$ for 5,000(UT)-2,000(V) and 15,000(UT)-2,000(V) 
capsules. Those values are in concurrence with the results obtained from optical microscopy and STEM images (Figure 1).
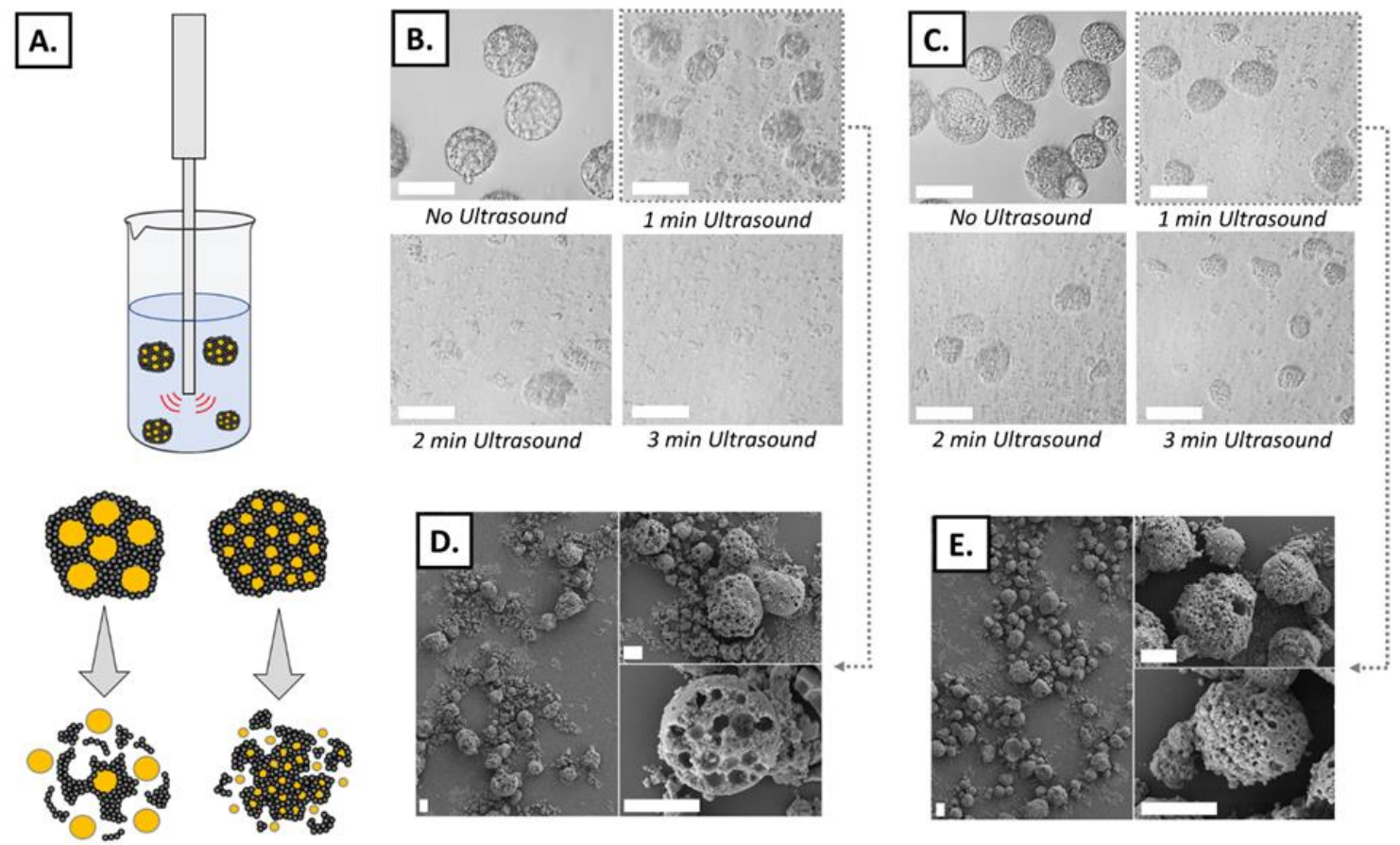

Figure 4. Rupture of dopant-free silica microcapsules using ultrasound. (A) Schematic of the sonication and rupturing process. Optical microscopy images of (B) 5,000 (UT) 2,000 (V) and (C) 15,000 (UT) - 2,000 (V) capsules after $0 \mathrm{~min}, 1 \mathrm{~min}, 2 \mathrm{~min}$ or $3 \mathrm{~min}$ of ultrasound. Scanning electron microscopy images of (D) 5,000 (UT) - 2,000 (V) and (E) $15,000(\mathrm{UT})-2,000(\mathrm{~V})$ capsules after $1 \mathrm{~min}$ of ultrasound. Scale bars are equal to 20 $\mu \mathrm{m}$ for optical microscopy images and $10 \mu \mathrm{m}$ for SEM images.

This ability to adjust the capsule robustness by simple modification of the internal structure makes the method promising for producing versatile capsules for use in triggered release applications. However, further work is required to quantify this aspect. For this, techniques such as Micromanipulation and Atomic Force Microscopy (AFM) could be considered. Low magnification optical microscopy images of samples after ultrasound and additional SEM images of capsules after 1 min of ultrasound are provided in the Supporting information (Figures S12 and S13). 


\subsection{Encapsulation of a hydrophobic compound}

Encapsulation of a hydrophobic dye (i.e. Nile red) was used as a simple tool to confirm the presence of oil cores within the capsule structure. A pink solid was visually observed following capsule cleaning, suggesting encapsulation of dye within the structure. Confocal microscopy images of Nile red loaded capsules taken $48 \mathrm{~h}$ after preparation exhibited bright pink spots (Figure 5). This observation confirms the presence of oil within the capsule, as well as the multicore structure, corroborating the results from STEM, SEM, and optical microscopy. These results suggest the ability of the system to entrain hydrophobic actives. However, further work is required to explore the encapsulation of diverse hydrophobic actives (e.g. vitamins, antioxidants, etc), and assess how the change of the capsule internal structure can impact the release of such encapsulated materials.
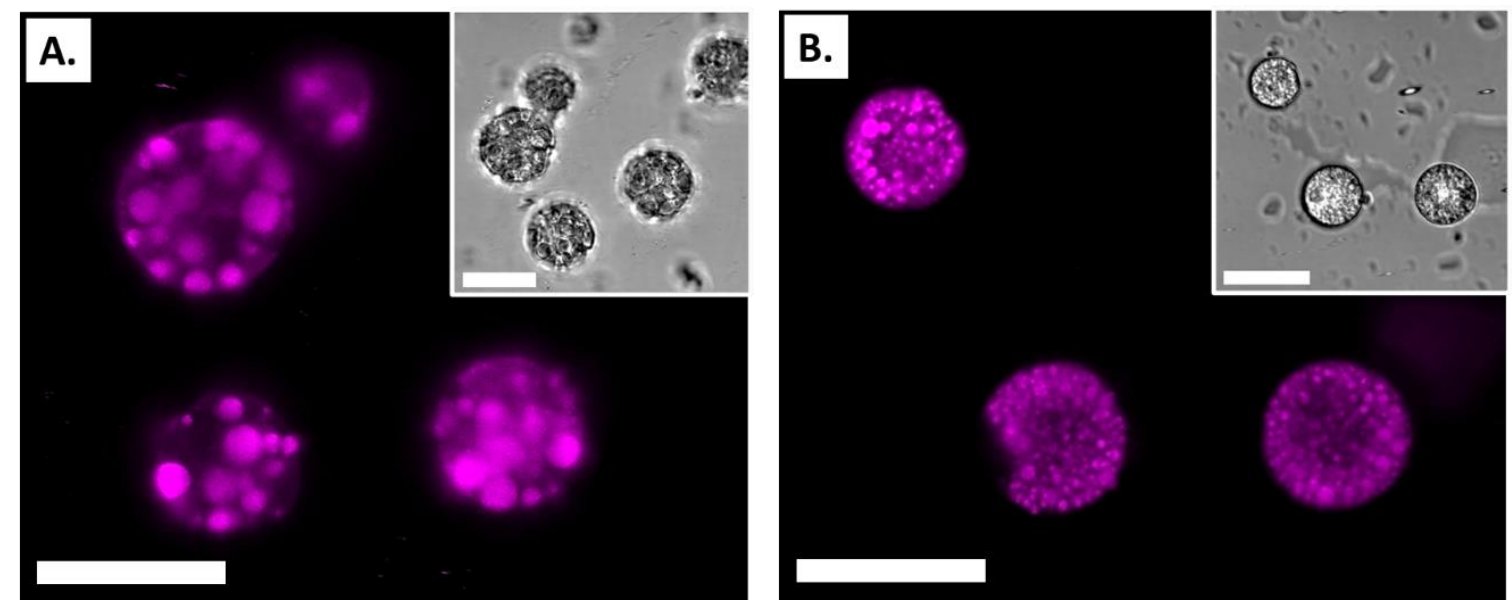

Figure 5. Confocal microscopy images of (A) 5,000 (UT)-2,000 (V) and (B) 15,000 (UT) $2,000(\mathrm{~V})$ capsules loaded with Nile red. Insets are the corresponding bright field images. Scale bars are equal to $20 \mu \mathrm{m}$.

\section{Conclusion}

This paper reports a novel method for producing multi-oil core silica colloidosomes via saltdriven assembly of nanoparticles in an Oil-in-Water-in-Oil emulsion template. In this approach, capsules are produced without requiring a Pickering emulsion intermediate step, enabling direct use of nanoparticles without any surface functionalization or surfactant adsorption. This approach offers multiple advantages compared to conventional encapsulation techniques. Firstly, the capsules are easy to produce with minimal lab equipment, offering exceptional promise for scalability. Secondly, the entire process can be undertaken at room temperature 
without harmful or toxic reagents, making the synthesis and final product environmentally friendly. Finally, the emulsion templating route provides a high degree of versatility for controlling the physicochemical properties of the final material. We demonstrate incorporation of $\mathrm{Fe}_{3} \mathrm{O}_{4}, \mathrm{TiO}_{2}$ and $\mathrm{ZnO}$ nanoparticles within the structure for specialised functionalities: magnetic removal and photocatalysis. This preparation method also offers a degree of control on the overall size of the capsules as well as the inner core dimensions by adjusting mixing conditions and system composition. These capsules were able to retain a hydrophobic dye (Nile red) within their structure and could be ruptured by ultrasound, enabling direct access to the inner cores. We believe this work showcases an effective procedure for producing a novel multifunctional colloidal material that can be tailored for a variety of industrial applications using cheap, commercially available nanoparticles as building blocks.

\section{Experimental section}

\section{Materials}

All chemicals were obtained from Sigma-Aldrich or Fisher scientific, except where otherwise specified, and were used without further purification: Sunflower oil (Sainsburys), Absolute ethanol, Ludox HS-40 ( $\mathrm{SiO}_{2}, 40$ wt\% in $\mathrm{H}_{2} \mathrm{O}, 15-25 \mathrm{~nm}$ diameter), Tween 80, Span 80, Calcium chloride dihydrate $\left(\mathrm{CaCl}_{2}, 2 \mathrm{H}_{2} \mathrm{O}\right)$, Nile Red (Dry powder, 99\%), Rhodamine B (Dry powder, 95\%), Iron (II, III) oxide $\left(\mathrm{Fe}_{3} \mathrm{O}_{4}\right.$, dry powder, 50 - $100 \mathrm{~nm}$ diameter), Titanium dioxide $\left(\mathrm{TiO}_{2}\right.$, dry powder, $21 \mathrm{~nm}$ diameter, 99.5\%) and Zinc oxide ( $\mathrm{ZnO}, 20$ wt\% in $\mathrm{H}_{2} \mathrm{O},<100 \mathrm{~nm}$ diameter). It is worth mentioning that a multi-component oil such as sunflower oil was preferred to monocomponents oils such as Linseed oil or mineral oils for practical and economic reasons. Sunflower oil is cheap, safe, and readily available, enabling its use as a proof of principle oil phase. Furthermore, a control experiment under UV-light irradiation confirmed that sunflower oil does not generate UV-active by-products, which enable its use in capsules considered for photocatalytic experiments. It is also worth mentioning that the Ludox HS40 was used 6-12 months after opening and exhibited a higher viscosity compared to freshly opened bottles. We believe this was likely due to progressive hydrolysis of the silica nanoparticle surface over time. Supplementary experiments confirmed that the same trends 
could be observed with fresh Ludox HS40 bottles. Adjusting the surfactant concentration serves to compensate for the difference in viscosity (Figures S14 to S17).

\section{Preparation of simple $\left(\mathrm{O}_{1} / \mathrm{W}\right)$ and double $\left(\mathrm{O}_{1} / \mathrm{W} / \mathrm{O}_{2}\right)$ emulsions}

The aqueous phase (W) was prepared by addition of silica nanoparticles (35 wt\%) and Tween $80\left(1,5\right.$ or 10 wt\%) to deionized water. The internal oil phase $\left(\mathrm{O}_{1}\right)$ was composed of sunflower oil. External oil phase $\left(\mathrm{O}_{2}\right)$ was composed of sunflower oil and Span 80 (1 wt\%). Primary emulsions were prepared by mixing the internal oil phase $(2 \mathrm{~g})$ with the aqueous phase $(8 \mathrm{~g})$ for 60 s using an Ultra Turrax mixer (T 25 digital, IKA) set at 3,000, 5,000, 10,000 or 15,000 RPM. Secondary emulsions were prepared by mixing the primary emulsion $(1 \mathrm{~g})$ and the external oil phase (9 g) for 60 s using a Vortex mixer (TopMix FB15024, Fischer Scientific) set at 2,000 RPM. The stability of different emulsions was assessed at regular time intervals using visual observation and optical microscopy.

\section{Preparation of multi-oil core silica capsules}

Oil core microcapsules were prepared from $\mathrm{O}_{1} / \mathrm{W} / \mathrm{O}_{2}$ double emulsions using the method presented in Scheme 1. The aqueous phase (W) was prepared by addition of $\mathrm{SiO}_{2}$ nanoparticles (35 wt\%) and Tween 80 (5 wt\%) to deionized water. For metal oxide doping experiments, $\mathrm{Fe}_{3} \mathrm{O}_{4}(5 \mathrm{wt} \%), \mathrm{TiO}_{2}(5 \mathrm{wt} \%)$ or $\mathrm{ZnO}(1.5 \mathrm{wt} \%)$ nanoparticles were also added to the aqueous phase before homogenization of the mixture for $5 \min (30 \mathrm{~s}$ on - $10 \mathrm{~s}$ off) using an ultrasonic homogenizer (Sonic Dismembrator FB-120, Fisher Scientific) operating at an frequency of $20 \mathrm{kHz}$ and an amplitude of $100 \%$. The internal oil phase $\left(\mathrm{O}_{1}\right)$ was composed of sunflower oil and the external oil phase $\left(\mathrm{O}_{2}\right)$ was composed of sunflower oil and Span 80 (1 wt\%). Primary emulsions were prepared by mixing the internal oil phase $(2 \mathrm{~g})$ with the aqueous phase (8 g) for 60 s using an Ultra Turrax mixer set at 5,000, 10,000 or 15,000 RPM. Secondary emulsions were prepared by mixing the primary emulsion $(1 \mathrm{~g})$ and the external oil phase $(9$ g) for $60 \mathrm{~s}$ using a using either a Vortex (V) or an Ultra Turrax (UT) mixer. Capsules were then formed after dropwise addition of a calcium chloride solution ( $\left.1 \mathrm{~mL}, 1 \mathrm{~mol} . \mathrm{L}^{-1}\right)$ into the $\mathrm{O}_{1} / \mathrm{W} / \mathrm{O}_{2}$ emulsion, whilst maintaining 2,000 RPM stirring with a Vortex mixer (V). The capsules were then recovered from solution via centrifugation (3,000 RPM, $5 \mathrm{~min})$, and cleaned twice by sequential redispersal in deionized water followed by centrifugation $(2,000$ RPM, 5 min). The cleaned capsules were finally redispersed in deionized water. 


\section{Structural characterisation of emulsions and capsules}

The internal structure, overall diameter and chemical composition of the capsules were assessed using optical and electron microscopy. Optical microscopy images of the aqueous dispersion of capsules were obtained using a Leica DME microscope equipped with 10x and $63 \times$ objective lenses. Scanning Electron Microscopy (SEM) images were taken using a Tescan Mira3 FEG-SEM microscope (acceleration voltage of $5 \mathrm{kV}$ ). Scanning transmission electron microscopy (STEM) images were obtained using a Talos F200X G2 from Thermo Scientific equipped with a Ceta $16 \mathrm{M}$ camera at an acceleration voltage of $200 \mathrm{kV}$. The same setup was used to examine the incorporation of metal oxides into the structure via Energy Dispersive Xray spectroscopy (EDS). SEM/STEM samples reported in this article were prepared via deposition and drying of an aqueous dispersion of the capsules on a SEM stub or a TEM membrane. The droplet and capsule diameters were obtained using ImageJ software (imagej.net), analyzing 250 randomly chosen droplets or capsules. Image analysis was mostly carried out on optical microscopy images, due to the low cost, high speed, and ease of this technique compared to electron microscopy techniques. All the reported values correspond to the mean plus or minus one standard deviation. This interval of confidence was preferred to the conventional 2 standard deviations due to the non-symmetrical distribution of the samples (i.e. Poisson distribution).

\section{Shell rupture and access to internal cores}

Rupture of capsules was instigated via ultrasonication of an aqueous dispersion ( $5 \mathrm{~mL}$ ) for up to 3 min using an ultrasonic homogenizer operating at a frequency of $20 \mathrm{kHz}$ and an amplitude of $100 \%$. Samples were then centrifuged $(5,000 \mathrm{RPM}-5 \mathrm{~min})$ and cleaned twice with absolute ethanol before being redispersed in $5 \mathrm{~mL}$ of deionized water and observed with optical and electron microscopy.

\section{Magnetic properties of $\mathrm{Fe}_{3} \mathrm{O}_{4}$ doped capsules}

The magnetic response of $\mathrm{Fe}_{3} \mathrm{O}_{4}$ doped silica microcapsules was assessed via exposure to an external magnetic field. For this, $12 \mathrm{~mL}$ of aqueous solution containing $20 \mathrm{mg}$. $\mathrm{L}^{-1}$ of $\mathrm{Fe}_{3} \mathrm{O}_{4}$ doped silica capsules was exposed to a magnetic field generated by a Rare-earth neodymium magnet ( $\mathrm{NiCuNi}, 19 \times 28.2 \mathrm{~mm}, 1.2 \mathrm{~T}$, E-magnets UK). Pictures were taken every 15 seconds to analyse the separation process. 
Photocatalytic properties of $\mathrm{TiO}_{2}$ and $\mathrm{ZnO}$ doped capsules

The photocatalytic properties of $\mathrm{TiO}_{2}$ and $\mathrm{ZnO}$ doped silica microcapsules were assessed via degradation of an aqueous solution of Rhodamine B solution under UV-light irradiation. Sample preparation involved $100 \mathrm{mg}$ of capsules being added to a Rhodamine B solution (50 $\mathrm{mL}, 10 \mathrm{mg} \cdot \mathrm{L}^{-1}$ ). The solutions were then exposed to UV-light (36 W, Light excitation: $365 \mathrm{~nm}$ ) without preliminary equilibration in dark. $5 \mathrm{~mL}$ of solution was collected after $0,10,20,30,40$, 50 and 60 minutes and centrifuged at 5,000 RPM for $5 \mathrm{~min}$. Absorbance of the supernatant was measured using a UV-visible spectrophotometer (Cary 60, Agilent technologies). The absorption maximum was observed to shift from 555 to $528 \mathrm{~nm}$, and therefore was not interpreted at a fixed wavelength but at the position of the maximum. The compacted capsules were then redispersed by manual agitation with the supernatant, and the mixture was reinjected into the original solution. This procedure was repeated at subsequent time intervals. Rhodamine $\mathrm{B}$ solution with and without $\mathrm{SiO}_{2}$ microcapsules were used as references. Each experimental series was duplicated to ensure reproducibility of results.

\section{Preparation and characterization of Nile red loaded capsules}

Capsules containing Nile red (150 mg. $\left.\mathrm{L}^{-1}\right)$ were prepared. Initially, primary emulsions were prepared using an Ultra-Turrax mixer operating at 5,000 or 15,000 RPM and secondary emulsions were prepared using a Vortex mixer operating at 2,000 RPM. The formed capsules were imaged using a Leica SP5 confocal microscope equipped with a 63x oil objective (Excitation wavelength: $514 \mathrm{~nm}$, Detector range: 530-730 nm).

\section{Acknowledgements}

The authors gratefully acknowledge $\operatorname{Dr}$ Heather Greer and Luca Mascheroni from the Department of Chemistry and the Department of Chemical Engineering and Biotechnology at the University of Cambridge for their help with STEM, EDS analysis and confocal microscopy experiments. The authors also thank Dan Toy and Eleftheria Diamanti, for their help with some of the initial experiments. The authors acknowledge the EPSRC Underpinning Multi-User Equipment Call (EP/P030467/1) for funding the Talos, used for STEM and EDS analysis. D.F.F.B. thanks the University of Cambridge for funding through a W.D. Armstrong PhD Scholarship. T.M.M. thanks the Ernest Oppenheimer Fund for funding through an Oppenheimer Research Fellowship. 


\section{References}

[1] Gibbs, B.F.; Kermasha, S.; Alli, I.; Mulligan, C.N. Encapsulation in the Food Industry: a Review. Int. J. Food Sci. Nutr. 1999, 50, 213-224.

[2] Gouin, S. Microencapsulation: Industrial Appraisal of Existing Technologies and Trends. Trends Food Sci. Technol. 2004, 15, 330-347.

[3] Carvalho, I.T.; Estevinho, B.N.; Santos, L. Application of Microencapsulated Essential Oils in Cosmetic and Personal Healthcare Products - a Review. Int. J. Cosmet. Sci. 2016, 38, 109-119.

[4] Casanova, F.; Santos, L. Encapsulation of Cosmetic Active Ingredients for Topical Application - a Review. J. Microencapsul. 2016, 33, 1-17.

[5] Hack, B.; Egger, H.; Uhlemann, J.; Henriet, M.; Wirth, W.; Vermeer, A.W.P.; Duff, D.G. Advanced Agrochemical Formulations Through Encapsulation Strategies? Chem. Ing. Tech. 2012, 84, 223-234.

[6] Tsuji, K. Microencapsulation of Pesticides and their Improved Handling Safety. J. Microencapsul. 2001, 18, 137-147.

[7] Singh, M.N.; Hemant, K.S.Y.; Ram, M.; Shivakumar, H.G. Microencapsulation: A Promising Technique for Controlled Drug Delivery. Res Pharm Sci. 2010, 5, 65-77.

[8] Ma, G. Microencapsulation of Protein Drugs for Drug Delivery: Strategy, Preparation, and Applications. J. Control Release. 2014, 193, 324-340.

[9] Yow, H.N.; Routh, A.F. Formation of Liquid Core-polymer Shell Microcapsules. Soft Matter. 2006, 2, 940-949.

[10] Dubey, R.; Shami, T.C.; Rao, K.U.B. Microencapsulation Technology and Applications. Def. Sci. J. 2009, 59, 82-95.

[11] Velev, O.D.; Furusawa, K.; Nagayama, K. Assembly of Latex Particles by Using Emulsion Droplets as Templates. 1. Microstructured Hollow Spheres. Langmuir. 1996, 12, 2374-2384.

[12] Dinsmore, A.D.; Hsu, M.F.; Nikolaides, M.G.; Marquez, M.; Bausch, A.R.; Weitz, D.A. Colloidosomes: Selectively Permeable Capsules Composed of Colloidal Particles. Science. 2002, 298, 1006-1009. 
[13] Rossier-Miranda, F.J.; Schroën, C.G.P.H.; Boom, R.M. Colloidosomes: Versatile Microcapsules in Perspective. Colloids and Surfaces A: Physicochem. Eng. Aspect. 2009, 343, 43-49.

[14] Thompson, K.L.; Williams, M.; Armes, S.P. Colloidosomes: Synthesis, Properties, and Applications. J. Colloid. Interf. Sci. 2015, 447, 217-228.

[15] Kim, J.W.; Fernandez-Nieves, A.; Dan, N.; Utada, A. S.; Marquez, M.; Weitz, D. A. Colloidal Assembly Route for Responsive Colloidosomes with Tunable Permeability. Nano Lett. 2007, 7, 2876-2880.

[16] Yow, H.N.; Routh, A.F. Release Profiles of Encapsulated Actives from Colloidosomes Sintered for Various Durations. Langmuir. 2009, 25, 159-166.

[17] Duan, H.; Wang, D.; Sobal, N.S.; Giersig, M.; Kurth, D.G.; Mohwald, H. Magnetic Colloidosomes Derived from Nanoparticle Interfacial Self-Assembly. Nano Lett. 2005, 5, 949952.

[18] Zhang, L.; Zhang, F.; Wang, Y.S.; Sun, Y.L.; Dong, W.F.; Song, J.F.; Huo, Q.S.; Sun, H.B. Magnetic Colloidosomes Fabricated by $\mathrm{Fe}_{3} \mathrm{O}_{4}-\mathrm{SiO}_{2}$ Hetero-nanorods. Soft Matter. 2011, 7, 7375-7381.

[19] Bollhorst, T.; Shahabi, S.; Worz, K.; Petters, C.; Dringen, R.; Maas, M.; Rezwan, K. Bifunctional Submicron Colloidosomes Coassembled from Fluorescent and Superparamagnetic Nanoparticles. Angew. Chem. 2015, 127, 120-125.

[20] Li, S.; Moosa, B.A.; Croissant, J.G.; Khashab, N.M. Electrostatic Assembly/Disassembly of Nanoscaled Colloidosomes for Light-triggered Cargo Release. Angew. Chem. 2015, 127, 69086912.

[21] Jiang, H.; Hong, L.; Li, Y.; Ngai, T. All-silica Submicrometer Colloidosomes for Cargo Protection and Tunable Release. Angew. Chem. Int. Ed. 2018, 57, 11836-11840.

[22] Cayre, O.J.; Hitchcock, J.; Manga, M.S.; Fincham, S.; Simoes, A.; Williams, R.A.; Biggs, S. pH-responsive Colloidosomes and their Use for Controlling Release. Soft Matter. 2012, 8, 4717-4724. 
[23] Zhou, S.; Fan, J.; Datta, S.S.; Guo, M.; Guo, X.; Weitz, D.A. Thermally Switched Release from Nanoparticle Colloidosomes. Adv. Funct. Mater. 2013, 23, 5925-5929.

[24] Sun, Q.; Du, Y.; Hall, E.A.H.; Luo, D.; Sukhorukov, G.B.; Routh, A.F. A Fabrication Method of Gold Coated Colloidosomes and their Application as Targeted Drug Carriers. Soft Matter. 2018, 14, 2594-2603.

[25] Pickering, S.U. Emulsions. J. Chem. Soc. Trans. 1907, 91, 2001-2021.

[26] Wu, J.; Ma, G.H. Recent Studies of Pickering Emulsions: Particles Make the Difference. Small. 2016, 12, 4633-4648.

[27] Binks, B.P. Particles as Surfactants-similarities and Differences. Curr. Opin. Colloid Interface Sci. 2002, 7, 21-41.

[28] Keen, P.H.R.; Slater, N.K.H.; Routh, A.F. Encapsulation of Yeast Cells in Colloidosomes. Langmuir. 2011, 28, 1169-1174.

[29] Keen, P.H.R.; Slater, N.K.H.; Routh, A.F. Encapsulation of Lactic Acid Bacteria in Colloidosomes. Langmuir. 2012, 28, 16007-16014.

[30] Keen, P.H.R.; Slater, N.K.H.; Routh, A.F. Encapsulation of Amylase in Colloidosomes. Langmuir. 2014, 30, 1939-1948.

[31] Sun, Q.; Gao, H.; Sukhorukov, G.B.; Routh, A.F. Silver-coated Colloidosomes as Carriers for an Anticancer Drug. ACS Appl. Mater. Inter. 2017, 9, 32599-32606.

[32] Rodriguez, A.M.B.; Binks, B.P. Capsules from Pickering Emulsion Templates. Curr. Opin. Colloid Interface Sci. 2019, 44, 107-129.

[33] Palamarchuk, K.V.; Bukreeva, T.V.; Kalashnikova, I.V.; Zelenkov, V.N.; Potapov, V.V. Encapsulation of Oil Phases with Different Compositions into a Shell Composed of Nanoparticles of Natural Hydrothermal Silica and Polyelectrolyte Layers. Colloid journal. 2021, $83,228-235$.

[34] Li, J.; Stover, H.D.H. Pickering Emulsion Templated Layer-by-layer Assembly for Making Microcapsules. Langmuir. 2010, 26, 15554-15560. 
[35] Yang, F.; Ma, S.; Zong, W.; Luo, N.; Lv, M.; Hu, Y.; Zhou, L.; Han, X. Fabrication of pH Sensitive Microcapsules using Soft Templates and their Application to Drug Release. RSC Adv. 2015, 5, 51271-51277.

[36] Leong, J.Y.; Tey, B.T.; Tan, C.P.; Chan, E.S. Nozzleless Fabrication of Oil-core Biopolymeric Microcapsules by the Interfacial Gelation of Pickering Emulsion Templates. ACS Appl. Mater. Interfaces. 2015, 7, 16169-16176.

[37] Tan, P.Y.; Tan, T.B.; Chang, H.W.; Mwangi, W.W.; Tey, B.T.; Chan, E.S.; Lai, O.M.; Liu, Y.; Wang, Y.; Tan, C.P. Pickering Emulsion-templated Ionotropic Gelation of Tocotrienol Microcapsules: Effects of Alginate and Chitosan Concentrations and Gelation Process Parameters. J. Sci. Food Agric. 2021, doi.org/10.1002/jsfa.11249.

[38] Thompson, K.L.; Armes, S.P.; Howse, J.R.; Ebbens, S.; Ahmad, I.; Zaidi, J.H.; York, D.W.; Burdis, J.A. Covalently Cross-linked Colloidosomes. Macromolecules. 2010, 43, 10466-10474.

[39] Williams, M.; Armes, S.P.; York, D.W. Clay-based colloidosomes. Langmuir. 2012, 28, 1142-1148.

[40] Walsh, A.; Thompson, K.L.; Armes, S.P.; York, D.W. Polyamine-functional Sterically Stabilized Latexes for Covalently Cross-linkable Colloidosomes. Langmuir. 2010, 26, 1803918048.

[41] Yi, H.; Deng, Y.; Wang, C. Pickering Emulsion-based Fabrication of Epoxy and Amine Microcapsules for Dual Core Self-Healing Coating. Compos. Sci Technol. 2016, 133, 51-59.

[42] Zhao, Y.; Li, Y.; Demco, D.E.; Zhu, X.; Moller, M. Microencapsulation of Hydrophobic Liquids in Closed All-silica Colloidosomes. Langmuir. 2014, 30, 4253-4261.

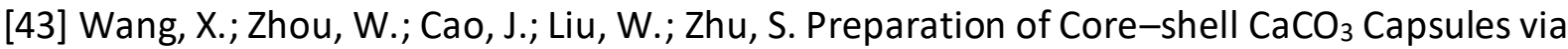
Pickering Emulsion Templates. J. Colloid Interface Sci. 2012, 372, 24-31.

[44] Stark, K.; Hitchcock, J.P.; Fiaz, A.; White, A.L.; Baxter, E.A.; Biggs, S.; McLaughan, J.R.; Freear, S.; Cayre, O.J. Encapsulation of Emulsion Droplets with Metal Shells for Subsequent Remote, Triggered Release. ACS Appl. Mater. Interfaces. 2019, 11, 12272-12282.

[45] Lee, D.; Weitz, D.A. Nonspherical Colloidosomes with Multiple Compartments from Double Emulsions. Small. 2009, 5, 1932-1935. 
[46] Brossault, D.F.F.; Routh, A.F. Salt-driven Assembly of Magnetic Silica Microbeads with Tunable Porosity. J. Colloid Interface Sci. 2020, 562, 381-390.

[47] Brossault, D.F.F.; McCoy, T.M.; Routh, A.F. Self-assembly of $\mathrm{TiO}_{2} / \mathrm{Fe}_{3} \mathrm{O}_{4} / \mathrm{SiO}_{2}$ Microbeads: A Green Approach to Produce Magnetic Photocatalysts. J. Colloid Interface Sci. 2021, 584, 779 788.

[48] Ma, Y.; Yao, J. Photodegradation of Rhodamine B Catalyzed by $\mathrm{TiO}_{2}$ Thin Films. J. Photochem. Photobiol. A. 1998, 116, 167-170.

[49] Watanabe, T.; Takizama, T.; Honda, K. Photocatalysis through Excitation of Adsorbates. 1. Highly Efficient N-deethylation of Rhodamine B Adsorbed to CdS. J. Phys. Chem. 1977, 81, 1845-1851.

[50] Guo, C.; Xu, J.; He, Y.; Zhang, Y.; Wang, Y. Photodegradation of Rhodamine B and Methyl Orange over One-dimensional $\mathrm{TiO}_{2}$ Catalysts under Simulated Solar Irradiation. Appl. Surf. Sci. 2011, 257, 3798-3803. 